\title{
GMR
}

\section{Prognostic significance of long non-coding RNA MALAT-1 in various human carcinomas: a meta-analysis}

\author{
J. Wang ${ }^{1 *}$, A.M. Xu ${ }^{1 *}$, J.Y. Zhang ${ }^{1 *}$, X.M. He ${ }^{2}$, Y.S. Pan ${ }^{1}$, G. Cheng ${ }^{1}$, C. Qin ${ }^{1}$, \\ L.X. Hua ${ }^{1}$ and Z.J. Wang ${ }^{1}$ \\ ${ }^{1}$ State Key Laboratory of Reproductive Medicine, Department of Urology, \\ First Affiliated Hospital of Nanjing Medical University, Nanjing, China \\ 2Department of Obstetrics and Gynecology, \\ First Affiliated Hospital of Nanjing Medical University, Nanjing, China \\ *These authors contributed equally to this study. \\ Corresponding author: L.X. Hua \\ E-mail: drhua1966@163.com
}

Genet. Mol. Res. 15 (1): gmr.15017433

Received August 12, 2015

Accepted November 9, 2015

Published January 29, 2016

DOI http://dx.doi.org/10.4238/gmr.15017433

ABSTRACT. The long non-coding RNA MALAT-1 plays an important role in cancer prognosis. The present research aimed to elucidate its precise predictive value in various human carcinomas. A quantitative meta-analysis was performed by searching PubMed, Embase, Web of Science, and Cochrane Library (most recently, January 2015) databases, and extracting data from studies that investigated the association between MALAT-1 expression and survival outcomes in patients of various cancers. Pooled hazard ratios (HRs) with $95 \%$ confidence intervals (Cls) were calculated as a measure of generalized effect. This meta-analysis included 1317 cases from 12 datasets. Our investigation revealed that poor overall survival (OS; $\mathrm{HR}=2.14,95 \% \mathrm{Cl}=1.74-2.64)$ and shortened disease-free, recurrencefree, disease-specific, or progression-free survival $(\mathrm{HR}=2.13,95 \% \mathrm{Cl}$ $=1.22-3.72$ ) can be predicted by high MALAT-1 expression for various cancers. Moreover, elevated MALAT-1 levels significantly correlated with 
decreased OS in a renal cell carcinoma (RCC) subgroup (HR $=3.43,95 \%$ $\mathrm{Cl}=1.80-6.53)$. These results imply that MALAT-1 can be used to predict unfavorable prognoses for several cancers, particularly RCC.

Key words: Long non-coding RNA; MALAT-1; Cancer; Prognosis; Survival

\section{INTRODUCTION}

Cancer is a multi-factorial disease and has become a major cause of morbidity and mortality in most countries (Bray et al., 2013). The five-year survival rate remains low for many cancers, and elaborate efforts have been made in the generation of reliable and persuasive evidence to identify prognostic biomarkers for carcinoma patients (Gonzalez and Agudo, 2012). However, efficient prognostic markers associated with high cancer mortality are lacking because of complex interactions between molecular abnormalities and cancer pathogenesis.

The discovery of long non-coding RNAs (IncRNAs) raised the prospect of their use in tumor diagnosis and therapy. The human genome sequencing project found that $70 \%$ of the human genome is transcribed, but only up to $2 \%$ comprises protein-coding sequences (Bertone et al., 2004; Kapranov et al., 2007). IncRNAs are endogenous transcripts greater than 200 nucleotides long and lack significant lengths of open reading frame (Chen and Carmichael, 2010). More importantly, influences on diverse biological processes, including cell development and pathogenesis, as well as prognosis, have been attributed to IncRNAs.

In recent years, several IncRNAs have been shown to participate in carcinogenesis and cancer progression (Gutschner and Diederichs, 2012). Metastasis-associated lung adenocarcinoma transcript 1 (MALAT-1) was initially recognized owing to its high expression in lung cancer tissue, and was identified as a prognostic factor for the survival of stage I non-small cell lung cancer (NSCLC) patients (Ji et al., 2003). Studies have revealed that MALAT-1 can promote cancer cell proliferation and metastasis by activating the ERK/MAPK pathway and interacting with hnRNP in cell cycle regulation (Yang et al., 2013; Wu et al., 2014). Moreover, research has demonstrated that high MALAT-1 expression can serve as an important prognostic element in other cancers such as pancreatic and colorectal malignancies and gliomas (Liu et al., 2014; Zheng et al., 2014; Ma et al., 2015).

However, most studies that have investigated the implications of MALAT-1 expression have been limited by small sample sizes and single tumor types. Therefore, we conducted a systematic review and quantitative meta-analysis to clarify the prognostic role of MALAT-1 expression in human cancers.

\section{MATERIAL AND METHODS}

\section{Search strategy}

This meta-analysis was performed in accordance with the guidelines of the Meta-analysis of Observational Studies in Epidemiology group (Stroup et al., 2000). We searched international databases (PubMed, Embase, Web of Science, and Cochrane) for relevant articles (most recently, January 19, 2015), using a strategy involving both Medical Subject Heading terms and free-text words to increase search sensitivity. The following key or text words were used: "long non-coding RNA", "IncRNA", "MALAT-1", "Metastasis-Associated-in-Lung-Adenocarcinoma-Transcript-1", "neoplasm", "cancer", "tumor", and "prognosis". Bibliographies of relevant reviews and included studies were also checked for other eligible investigations. 
Prognostic value of MALAT-1 in human cancers

\section{Study selection}

The following inclusion criteria were used: studies had to 1) involve patients with any type of carcinoma; 2) determine MALAT-1 expression levels in human tissues by quantitative realtime polymerase chain reaction (qRT-PCR) or microarray expression analysis or other methods; 3) investigate the relationship between MALAT-1 expression and survival outcome; 4) include a hazard ratio (HR) or survival curve for overall survival (OS) or time of recurrence, and an available or calculable $95 \%$ confidence interval $(\mathrm{Cl}$; if data were published in more than one article, only the most recent article was included); and 5) consist of full-text articles in English. The following were excluded: 1) reviews, letters, or laboratory studies lacking original data; 2) studies with overlapping or duplicate data; 3) investigations of non-dichotomous MALAT-1 expression levels and those lacking survival outcome; and 4) articles published in languages other than English.

\section{Data extraction}

Data from all eligible studies were independently extracted by two investigators (J.W. and A.M.X.) and comprised the first author's name, year of publication, study population, nationality, median or mean age of patients, ethnicity, type of disease and sample used, detection method, cutoff definition, follow-up time, and HRs associated with high MALAT-1 expression for OS, recurrencefree survival (RFS), disease-free survival (DFS), disease-specific survival (DSS), or progressionfree survival (PFS), along with their $95 \%$ Cls and $P$ values. If only Kaplan-Meier curves were available, we reconstructed HR estimates by extracting several survival rates at specified times from the survival curves, using previously reported methods (Williamson et al., 2002; Tierney et al., 2007). To reduce observer variation, data from Kaplan-Meier survival curves were independently evaluated by three researchers (J.W., A.M.X., and J.Y.Z.) using Engauge Digitizer version 4.1. All the above-mentioned data are presented in detail in Tables 1 and 2.

\section{Statistical analysis}

Statistical heterogeneity was assessed by visual inspection of forest plots, the chi-square test (and assessment of associated $P$ values) and Higgins's $l^{2}$. When $P$ values were less than 0.05 and/or $l^{2}$ exceeded $50 \%$, indicating the presence of heterogeneity, a random-effect model (the DerSimonian-Laird method) was used. Otherwise, a fixed-effect model (the Mantel-Haenszel method) was employed. Moreover, we minimized the influence of heterogeneity by classifying the studies included into subgroups based on similar characteristics. Sensitivity analysis was performed, and Galbraith plots were generated to identify the source of heterogeneity. Publication bias was estimated by visually assessing the asymmetry of an inverted funnel plot. Additionally, Begg's funnel plot test and Egger's linear regression test were performed to provide quantitative evidence of publication bias. $\mathrm{P}$ values $<0.05$ were considered statistically significant. All analyses were performed using Stata version 12.0 (StataCorp, College Station, TX, USA).

\section{RESULTS}

\section{Characteristics of eligible studies}

As shown in Figure 1, ninety-six records relating to MALAT-1 expression and human cancer were identified in PubMed, Embase, Web of Science, and Cochrane databases. After 
reviewing the abstracts, 76 studies were excluded because they were irrelevant to the current analysis, were letters, reviews, or duplicate studies, or were not published in English. Furthermore, eight potentially suitable works were excluded as they lacked sufficient survival data (HRs and survival curves) for analysis. Therefore, 12 eligible articles were included in this meta-analysis (Ji et al., 2003; Schmidt et al., 2011; Lai et al., 2012; Cho et al., 2014; Liu et al., 2014; Okugawa et al., 2014; Zheng et al., 2014; Hirata et al., 2015; Ma et al., 2015; Pang et al., 2015; Shen et al., 2015; Zhang et al., 2015).

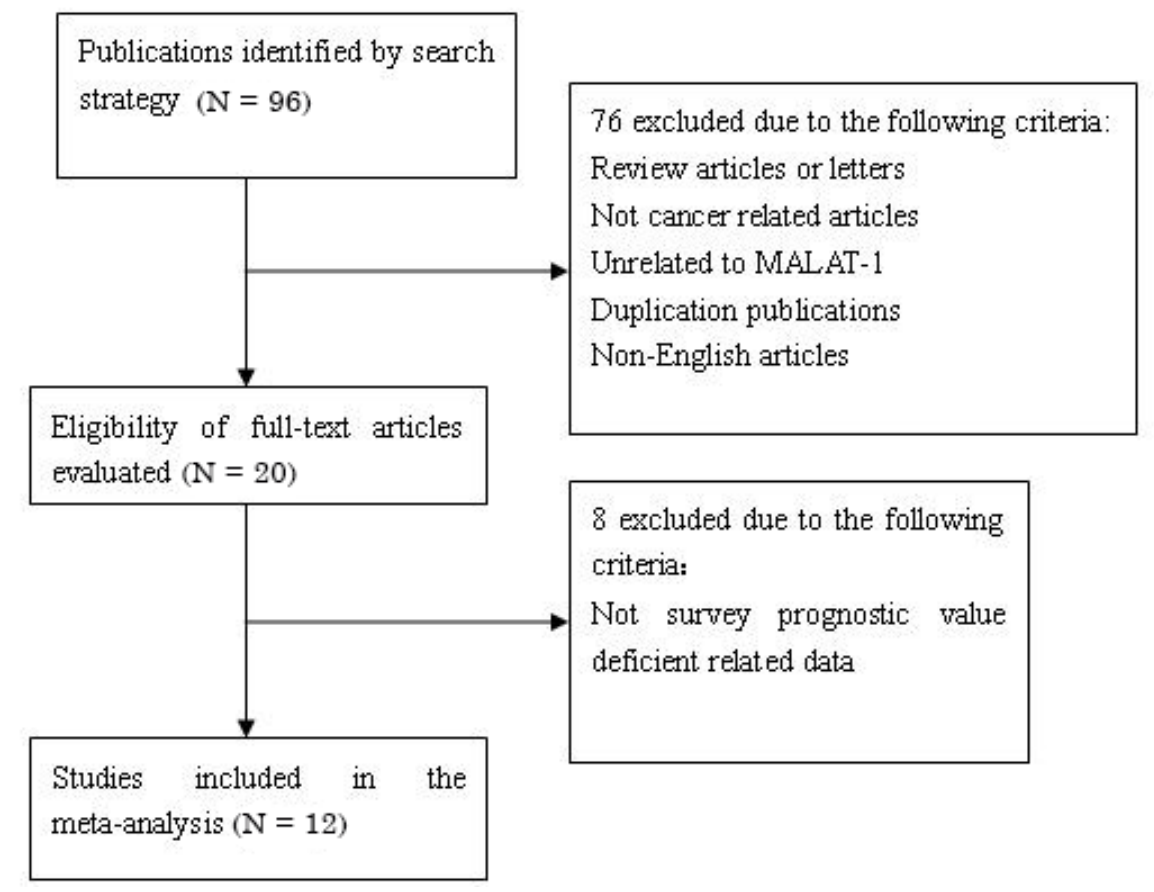

Figure 1. Flow diagram of the study selection process.

The main features and results of the eligible articles are summarized in Tables 1 and 2 . These studies investigated a total of 1337 cases from China, Japan, and Germany. The patients were classified as either Asian or Caucasian according to their ethnic background, with 10 articles involving the former and two the latter. Ten studies used only one measure of survival. Of these, seven focused on OS, while DFS, DSS, and RFS were used separately in three investigations. One report tested both OS and PFS, while another calculated OS and DFS. All selected studies were published within the last five years with one exception, which was published more than 10 years ago. Regarding the method used to detect MALAT-1 expression, nine studies utilized qRTPCR, one performed in situ hybridization, one conducted microarray analysis, and one employed subtractive hybridization. The cancers under investigation in these studies included renal cell carcinoma (RCC), gastric cancer, multiple myeloma, pancreatic cancer, NSCLC, glioma, colorectal cancer, hepatocellular carcinoma (HCC), and pancreatic ductal adenocarcinoma. MALAT-1 expression levels were measured in tumor tissues in all articles except one. The mean or median length of follow-up among these studies ranged from 40 to 416.67 months. 
Prognostic value of MALAT-1 in human cancers

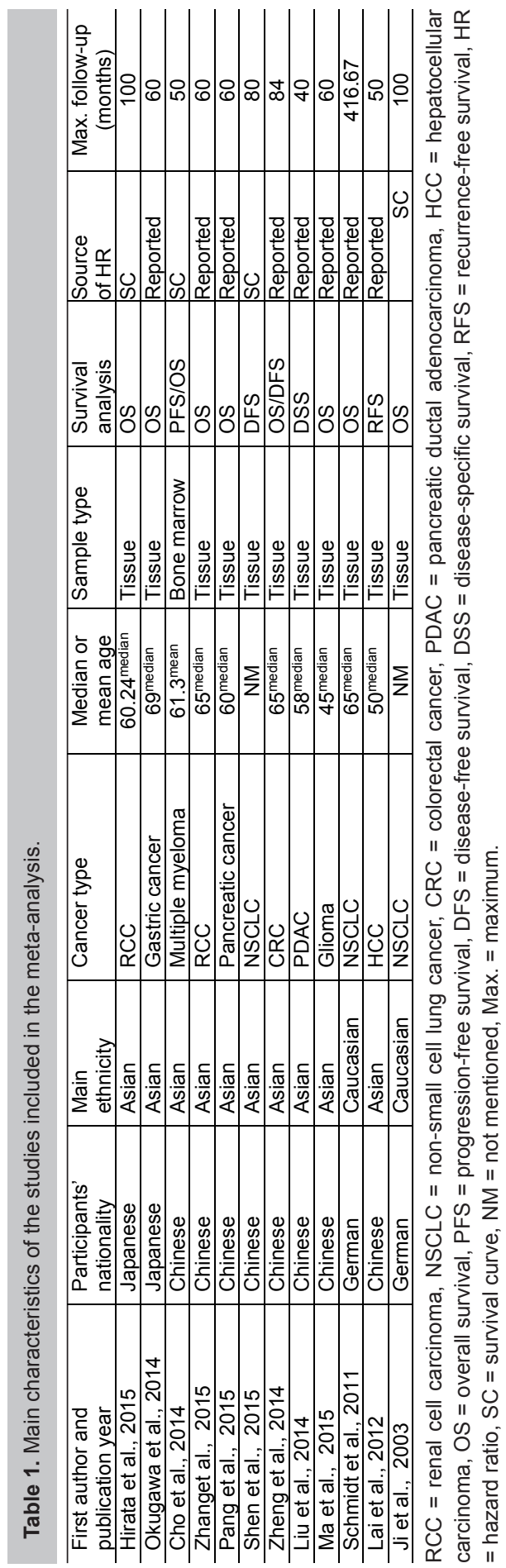




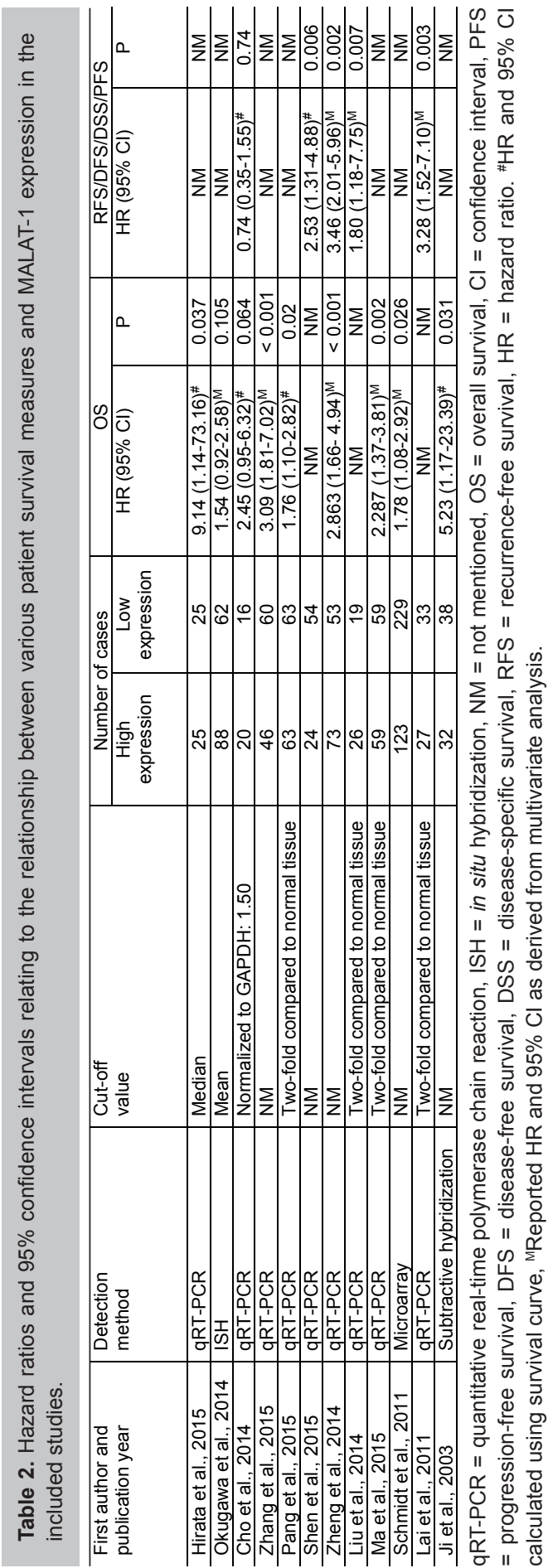




\section{Correlation between MALAT-1 expression and OS}

Among the nine articles evaluating OS, no statistically significant heterogeneity was evident $\left(I^{2}=4.3 \%, \mathrm{P}=0.399\right)$. Therefore, a fixed-effect model was used to calculate the pooled $\mathrm{HR}$ and $95 \%$ $\mathrm{Cl}$. The results showed that MALAT-1 expression was significantly associated with poor OS outcome in various carcinomas, with a pooled HR of $2.14(95 \% \mathrm{Cl}=1.74-2.64$; Figure 2$)$. The effect of MALAT-1 expression was further evaluated via subgroup analysis based on the cancer types investigated in those studies having measured OS. Upregulation of MALAT-1 was significantly associated with worse OS in RCC (HR = 3.43, 95\% Cl = 1.80-6.53). By contrast, no significant relationship was observed in the NSCLC subgroup ( $\mathrm{HR}=2.40,95 \% \mathrm{Cl}=0.93-6.17$; Figure 3 ).

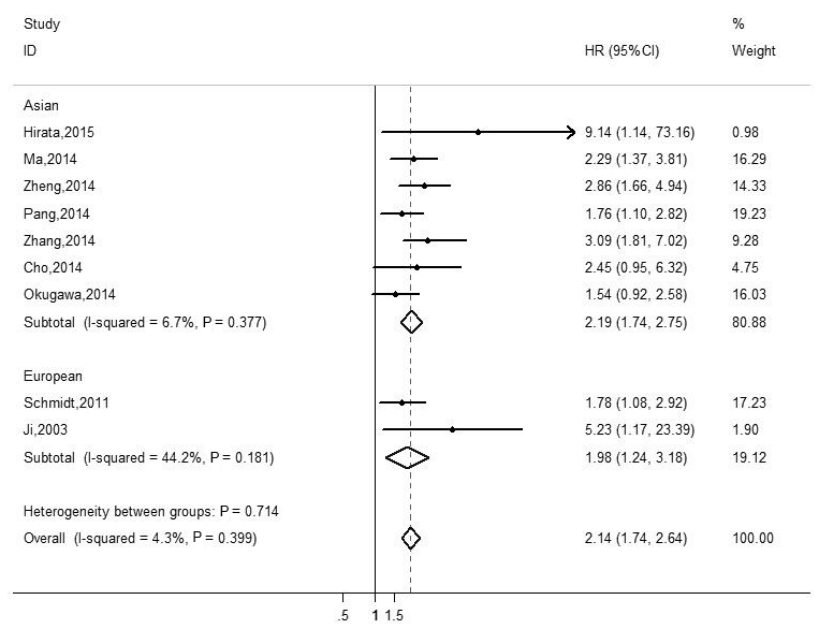

Figure 2. Forest plot summarizing the association between MALAT-1 expression and overall survival in patients with various cancers. $\mathrm{HR}=$ hazard ratio, $\mathrm{Cl}=$ confidence interval.

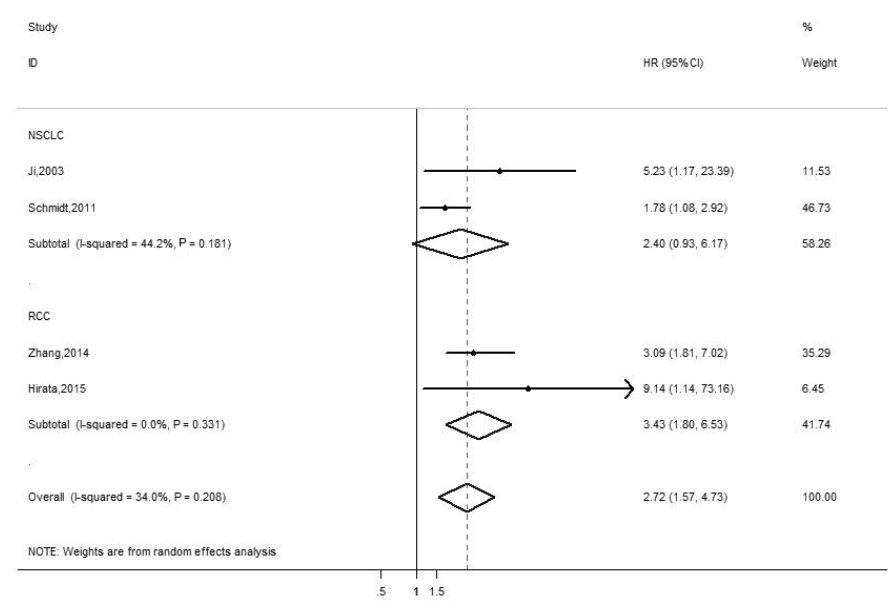

Figure 3. Forest plots representing subgroup analyses of the relationship between overall survival and MALAT-1 expression in different cancer types. $\mathrm{HR}=$ hazard ratio, $\mathrm{Cl}=$ confidence interval, $\mathrm{NSCLC}=$ non-small cell lung cancer, $\mathrm{RCC}=$ renal cell carcinoma. 


\section{Correlation between MALAT-1 expression and other survival measures}

As statistically significant heterogeneity was apparent among the five studies included in the DFS/RFS/DSS/PFS analysis $\left(I^{2}=67.3 \%, \mathrm{P}=0.016\right)$, we used a random-effects model to pool HRs. MALAT-1 overexpression was found to be significantly associated with poor DFS/RFS/DSS/ $\mathrm{PFS}$ in various cancers, with a pooled $\mathrm{HR}$ of $2.13(95 \% \mathrm{Cl}=1.22-3.72)$. However, these results also identified one study (Cho et al., 2014) as a possible source of the observed heterogeneity (Figure 4).

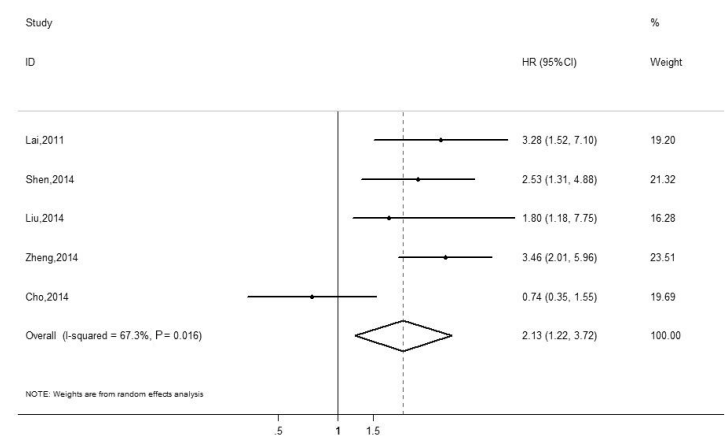

Figure 4. Forest plot of studies having assessed the association between expression of MALAT-1 and disease-, recurrence-, progression-free, and disease-specific survival of patients with various cancers. $\mathrm{HR}=\mathrm{hazard}$ ratio, $\mathrm{Cl}=$ confidence interval.

\section{Heterogeneity analysis}

Sensitivity analysis was performed by sequential omission of individual studies using the fixed-effect model. This test suggested that the Cho et al. (2014) study had influenced the overall result of the DFS/RFS/DSS/PFS analysis (Figures 5A and 5B). When the Galbraith plot was assessed, the same investigation (Cho et al., 2014) was also identified as an outlier causing heterogeneity in the analysis of survival measures other than OS (Figures 5C and 5D). By excluding this study, a similar and significant pooled $\mathrm{HR}$ was obtained $(\mathrm{HR}=2.88,95 \% \mathrm{Cl}=2.05-4.06)$. In addition, heterogeneity was found to be absent $\left(P=0.647, I^{2}=0.0 \%\right.$; Figure 6$)$.
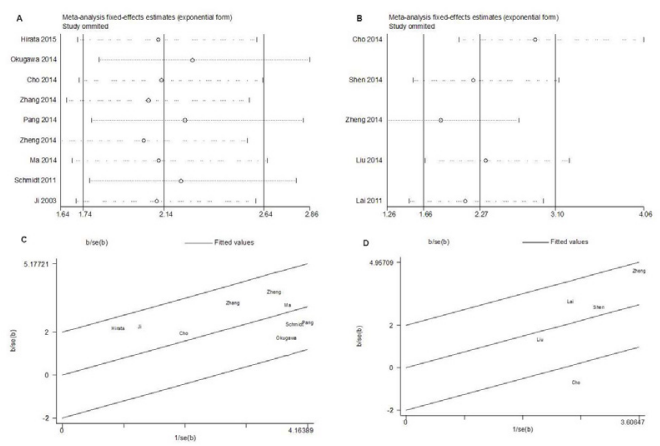

Figure 5. Sensitivity analyses and Galbraith radial plots for each analysis conducted. SE = standard error. A. Sensitivity analysis of studies having measured overall survival (OS). B. Sensitivity analysis of studies having measured disease-, recurrence-, progression-free, or disease-specific survival (DFS, RFS, PFS, and DSS, respectively). C. Galbraith radial plot of studies having measured OS. D. Galbraith radial plot of studies having measured DFS, RFS, PFS, or DSS. 


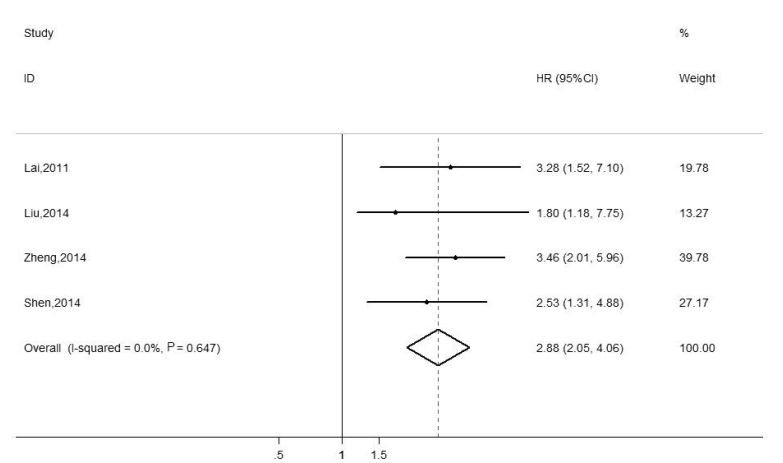

Figure 6. Forest plot of studies having evaluated the association between expression of MALAT-1 and disease-, recurrence-, progression-free, or disease-specific survival of patients with various cancers, following exclusion of data from Cho et al. (2014). HR = hazard ratio, $\mathrm{Cl}=$ confidence interval.

\section{Publication bias}

Begg's funnel plots and the Egger test were used to evaluate publication bias (Figures 7A and 7B). For the pooled analyses of OS and DFS/RFS/DSS/PFS, the Egger test returned $P$ values of 0.23 and 0.418 , respectively, and no obvious asymmetry was discerned in the corresponding funnel plots. Hence, this meta-analysis was free of any apparent publication bias.
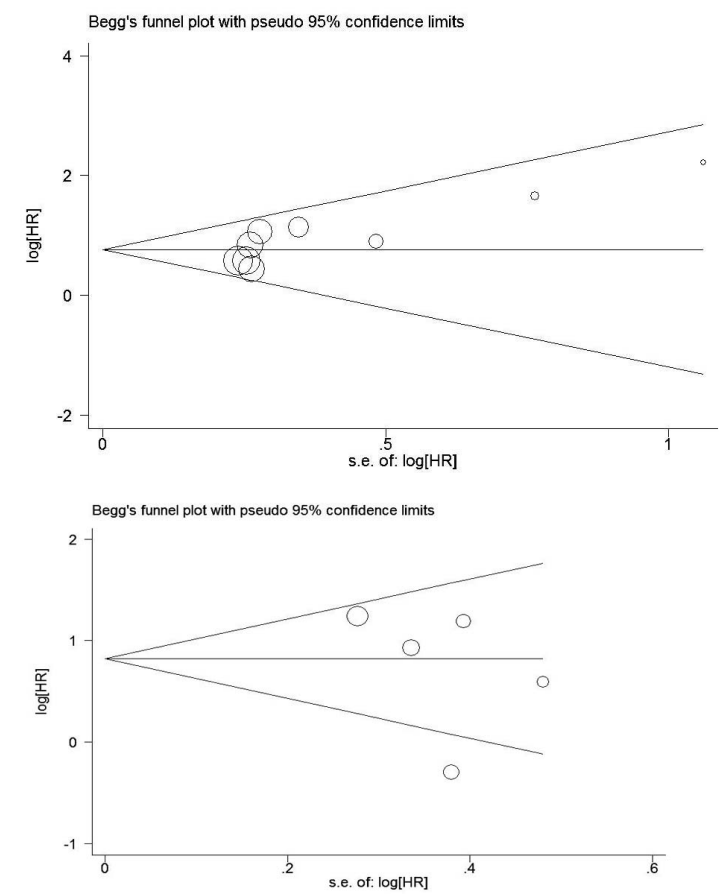

Figure 7. Funnel plots used to assess publication bias. $\mathrm{HR}=$ hazard ratio, $\mathrm{SE}=$ standard error. $\mathbf{A}$. Begg's funnel plot relating to analysis of overall survival. B. Begg's funnel plot relating to analysis of disease-, recurrence-, progressionfree, and disease-specific survival. 


\section{DISCUSSION}

In recent years, an increasing number of studies have focused on the role of IncRNAs in tumorigenesis and cancer outcomes. Some IncRNAs, such as HOX antisense intergenic RNA, prostate-specific transcript 1, cancer upregulated drug resistant, and IncRNA associated with microvascular invasion in HCC have been shown to participate in typical cancer processes, including progression and metastasis (Gutschner and Diederichs, 2012; Yuan et al., 2012). Importantly, IncRNAs have been considered potential biomarkers of cancer prognosis, given their unique expression profiles in the tissues or sera of cancer patients.

MALAT-1, also known as HCN, NEAT2, and PRO2853, is located on chromosome 11q13.1 and encodes a polyadenylated ncRNA of approximately 8 kb (Hutchinson et al., 2007). First established as a prognostic marker of lung cancer metastasis, it has also been linked to several other human tumors (Gutschner et al., 2013). In previous studies, MALAT-1 was found to be abnormally expressed in a variety of different human cancers, and highly conserved across several species, underscoring its functional importance (Yamada et al., 2006; Tano et al., 2010; Xu et al., 2011; Lai et al., 2012). Moreover, it has been shown to be upregulated in metastatic bladder cancer tissues and to contribute to bladder cancer cell migration (Ying et al., 2012). More importantly, MALAT-1 is an independent prognostic factor for HCC recurrence after liver transplant, and can interact with microRNAs to promote aggressive RCC (Lai et al., 2012; Hirata et al., 2015). However, the results of these studies are inconsistent, which may be attributed to differences in cancer types, patient ethnicities, and sample sources. To our knowledge, few studies have systematically evaluated the role of MALAT-1 expression in predicting clinical outcomes. Therefore, we conducted this metaanalysis to explore the prognostic value of MALAT-1 in survival and disease recurrence.

In this meta-analysis, we investigated the prognostic role of MALAT-1 in multiple human cancers, and included studies from Asian and Western countries to enhance its universal applicability to some extent. In addition, subgroup analyses using fixed- or random-effect models and sensitivity analysis were performed to optimize its statistical power to detect the role played by MALAT-1 in various cancers.

Upregulation of MALAT-1 correlated with poor survival rates (both OS and DFS/RFS/DSS/ PFS) in patients suffering from a range of cancers in this comprehensive investigation of 1337 cases from 12 datasets. Our OS analysis revealed a pooled HR of $2.14(95 \% \mathrm{Cl}=1.74-2.64)$, implying that increased MALAT-1 expression is associated with poor prognosis. Subsequently, the pooled result of the free survival analysis also indicated that increased MALAT-1 expression is predictive of worse outcome, with an $\mathrm{HR}$ of $2.13(95 \% \mathrm{Cl}=1.22-3.72)$. These significant findings suggest that MALAT-1 may serve as a novel independent predictive factor for poor prognosis in several cancers. Consequently, the current study provides new and strong evidence for the important role of MALAT-1 in the development and progression of various human tumors.

In addition, subgroup analyses based on cancer type revealed a statistically significant relationship with OS in the RCC subgroup, with a pooled HR of $3.43(95 \% \mathrm{Cl}=1.80-6.53)$. However, within the NSCLC subgroup, no such association was observed (pooled $\mathrm{HR}=2.40,95 \% \mathrm{Cl}=$ 0.93-6.17). This finding was intriguing, as when considered independently, the included NSCLC studies both demonstrated that elevated MALAT-1 expression can indicate poor prognosis, but a pooled analysis of the combined data returned no statistically significant result. This finding may be attributed to differences in tumor stage and classification, publication year, and statistical methods used. Overall, these results suggest that the detection of MALAT-1 expression in RCC patients may be valuable in the determination of prognoses. 
Following sensitivity analysis, one study (Cho et al., 2014) was identified as an outlier in the DFS/RFS/DSS/PFS assessment. When we excluded these data, heterogeneity was no longer evident $\left(I^{2}=0.0 \%, \mathrm{P}=0.647\right)$. After careful consideration, we concluded that potential sources of heterogeneity in this article may derive from the following factors. 1) Different diagnosis method. In this study, diagnoses of multiple myeloma were confirmed by bone marrow analyses showing monoclonal plasma cell counts over $10 \%$ and related laboratory tests. 2) Different sample storage conditions. In this research, isolated bone marrow samples were stored at $-80^{\circ} \mathrm{C}$ until RNA extraction. 3) Different RNA source. In this investigation, RNA was extracted from bone marrow samples. 4) The included studies differed in the qRT-PCR parameters and systems used, possibly affecting the results. 5) The cut-off value used to determine categories of MALAT-1 expression may also account for some inter-study heterogeneity.

Although the predictive value of MALAT-1 was statistically supported by this metaanalysis, our conclusions should be carefully interpreted for the following reasons. 1) The cut-off values deciding high and low MALAT-1 expression varied between studies. A unanimous value would be relatively difficult to achieve. 2) Post-operative treatment protocols differed among the diverse studies included, and these differences may have significantly influenced patient survival, resulting in some heterogeneity. 3) The statistical power of the association between MALAT-1 and DFS/RFS/DSS/PFS was reduced as a result of the relatively small sample size of 365 patients included in the five studies used in this particular meta-analysis. Thus, larger studies of improved design should be conducted to verify our results. 4) Some HRs could not be directly obtained from the primary studies, requiring us to extract HR estimates from survival curves. 5) We only included papers written in the English language. 6) Most of the included studies reported positive results, as those with negative findings are generally less likely to be published. Therefore, our results may overestimate the predictive significance of MALAT-1 in cancer prognosis to some extent.

In conclusion, this meta-analysis summarized current research on the relationship between aberrant MALAT-1 expression and cancer patient prognosis. Furthermore, it clarified that upregulation of MALAT-1 is significantly associated with poor survival in patients with various carcinomas, particularly RCC. MALAT-1 expression may serve as a novel predictive factor for poor prognosis in different cancer types. However, further investigation of the relationship between MALAT-1 levels and cancer prognosis is required to enable future clinical applications concerning this gene.

\section{Conflicts of interest}

The authors declare no conflict of interest.

\section{REFERENCES}

Bertone P, Stolc V, Royce TE, Rozowsky JS, et al. (2004). Global identification of human transcribed sequences with genome tiling arrays. Science 306: 2242-2246.http://dx.doi.org/10.1126/science.1103388

Bray F, Ren JS, Masuyer E and Ferlay J (2013). Global estimates of cancer prevalence for 27 sites in the adult population in 2008. Int. J. Cancer 132: 1133-1145.http://dx.doi.org/10.1002/ijc.27711

Chen LL and Carmichael GG (2010). Long noncoding RNAs in mammalian cells: what, where, and why? Wiley Interdiscip. Rev. RNA 1: 2-21.

Cho SF, Chang YC, Chang CS, Lin SF, et al. (2014). MALAT1 long non-coding RNA is overexpressed in multiple myeloma and may serve as a marker to predict disease progression. BMC Cancer 14: 809.http://dx.doi.org/10.1186/1471-2407-14-809

González CA and Agudo A (2012). Carcinogenesis, prevention and early detection of gastric cancer: where we are and where we should go. Int. J. Cancer 130: 745-753.http://dx.doi.org/10.1002/ijc.26430

Gutschner T and Diederichs S (2012). The hallmarks of cancer: a long non-coding RNA point of view. RNA Biol. 9: 703-719. http://dx.doi.org/10.4161/rna.20481 
Gutschner T, Hämmerle M and Diederichs S (2013). MALAT1 - a paradigm for long noncoding RNA function in cancer. J. Mol. Med. 91: 791-801.http://dx.doi.org/10.1007/s00109-013-1028-y

Hirata H, Hinoda Y, Shahryari V, Deng G, et al. (2015). Long noncoding RNA MALAT1 promotes aggressive renal cell carcinoma through Ezh2 and interacts with miR-205. Cancer Res. 75: 1322-1331.http://dx.doi.org/10.1158/0008-5472.CAN-14-2931

Hutchinson JN, Ensminger AW, Clemson CM, Lynch CR, et al. (2007). A screen for nuclear transcripts identifies two linked noncoding RNAs associated with SC35 splicing domains. BMC Genomics 8: 39.http://dx.doi.org/10.1186/1471-2164-8-39

Ji P, Diederichs S, Wang W, Böing S, et al. (2003). MALAT-1, a novel noncoding RNA, and thymosin beta4 predict metastasis and survival in early-stage non-small cell lung cancer. Oncogene 22: 8031-8041.http://dx.doi.org/10.1038/sj.onc.1206928

Kapranov P, Cheng J, Dike S, Nix DA, et al. (2007). RNA maps reveal new RNA classes and a possible function for pervasive transcription. Science 316: 1484-1488.http://dx.doi.org/10.1126/science.1138341

Lai MC, Yang Z, Zhou L, Zhu QQ, et al. (2012). Long non-coding RNA MALAT-1 overexpression predicts tumor recurrence of hepatocellular carcinoma after liver transplantation. Med. Oncol. 29: 1810-1816. http://dx.doi.org/10.1007/s12032-011-0004-z

Liu JH, Chen G, Dang YW, Li CJ, et al. (2014). Expression and prognostic significance of IncRNA MALAT1 in pancreatic cancer tissues. Asian Pac. J. Cancer Prev. 15: 2971-2977.http://dx.doi.org/10.7314/APJCP.2014.15.7.2971

Ma KX, Wang HJ, Li XR, Li T, et al. (2015). Long noncoding RNA MALAT1 associates with the malignant status and poor prognosis in glioma. Tumour Biol. 36: 3355-3359.http://dx.doi.org/10.1007/s13277-014-2969-7

Okugawa Y, Toiyama Y, Hur K, Toden S, et al. (2014). Metastasis-associated long non-coding RNA drives gastric cancer development and promotes peritoneal metastasis. Carcinogenesis 35: 2731-2739. http://dx.doi.org/10.1093/carcin/bgu200

Pang EJ, Yang R, Fu XB and Liu YF (2015). Overexpression of long non-coding RNA MALAT1 is correlated with clinical progression and unfavorable prognosis in pancreatic cancer. Tumour Biol. 36: 2403-2407. http://dx.doi.org/10.1007/s13277-014-2850-8

Schmidt LH, Spieker T, Koschmieder S, Schäffers S, et al. (2011). The long noncoding MALAT-1 RNA indicates a poor prognosis in non-small cell lung cancer and induces migration and tumor growth. J. Thorac. Oncol. 6: 1984-1992. http://dx.doi.org/10.1097/JTO.0b013e3182307eac

Shen L, Chen L, Wang Y, Jiang X, et al. (2015). Long noncoding RNA MALAT1 promotes brain metastasis by inducing epithelial-mesenchymal transition in lung cancer. J. Neurooncol. 121: 101-108. http://dx.doi.org/10.1007/s11060-014-1613-0

Stroup DF, Berlin JA, Morton SC, Olkin I, et al. (2000). Meta-analysis of observational studies in epidemiology: a proposal for reporting. Meta-analysis Of Observational Studies in Epidemiology (MOOSE) group. JAMA 283: 2008-2012. http://dx.doi.org/10.1001/jama.283.15.2008

Tano K, Mizuno R, Okada T, Rakwal R, et al. (2010). MALAT-1 enhances cell motility of lung adenocarcinoma cells by influencing the expression of motility-related genes. FEBS Lett. 584: 4575-4580.http://dx.doi.org/10.1016/j.febslet.2010.10.008

Tierney JF, Stewart LA, Ghersi D, Burdett S, et al. (2007). Practical methods for incorporating summary time-to-event data into meta-analysis. Trials 8: 16.http://dx.doi.org/10.1186/1745-6215-8-16

Williamson PR, Smith CT, Hutton JL and Marson AG (2002). Aggregate data meta-analysis with time-to-event outcomes. Stat. Med. 21: 3337-3351.http://dx.doi.org/10.1002/sim.1303

Wu XS, Wang XA, Wu WG, Hu YP, et al. (2014). MALAT1 promotes the proliferation and metastasis of gallbladder cancer cells by activating the ERK/MAPK pathway. Cancer Biol. Ther. 15: 806-814.http://dx.doi.org/10.4161/cbt.28584

Xu C, Yang M, Tian J, Wang X, et al. (2011). MALAT-1: a long non-coding RNA and its important $3 \phi$ end functional motif in colorectal cancer metastasis. Int. J. Oncol. 39: 169-175.

Yamada K, Kano J, Tsunoda H, Yoshikawa H, et al. (2006). Phenotypic characterization of endometrial stromal sarcoma of the uterus. Cancer Sci. 97: 106-112.http://dx.doi.org/10.1111/j.1349-7006.2006.00147.x

Yang F, Yi F, Han X, Du Q, et al. (2013). MALAT-1 interacts with hnRNP C in cell cycle regulation. FEBS Lett. 587: $3175-3181$. http://dx.doi.org/10.1016/j.febslet.2013.07.048

Ying L, Chen Q, Wang Y, Zhou Z, et al. (2012). Upregulated MALAT-1 contributes to bladder cancer cell migration by inducing epithelial-to-mesenchymal transition. Mol. Biosyst. 8: 2289-2294.http://dx.doi.org/10.1039/c2mb25070e

Yuan SX, Yang F, Yang Y, Tao QF, et al. (2012). Long noncoding RNA associated with microvascular invasion in hepatocellular carcinoma promotes angiogenesis and serves as a predictor for hepatocellular carcinoma patients' poor recurrence-free survival after hepatectomy. Hepatology 56: 2231-2241.http://dx.doi.org/10.1002/hep.25895

Zhang HM, Yang FQ, Chen SJ, Che J, et al. (2015). Upregulation of long non-coding RNA MALAT1 correlates with tumor progression and poor prognosis in clear cell renal cell carcinoma. Tumour Biol. 36: 2947-2955. http://dx.doi.org/10.1007/s13277-014-2925-6

Zheng HT, Shi DB, Wang YW, Li XX, et al. (2014). High expression of IncRNA MALAT1 suggests a biomarker of poor prognosis in colorectal cancer. Int. J. Clin. Exp. Pathol. 7: 3174-3181. 